\title{
ATG7 Polymorphisms rs7625184 and rs2606750 are Not Associated with Parkinson's Disease: A Case Control Study
}

\section{Ming Zou}

Shandong University

Jian-Yong Wang

Second Affiliated Hospital \& Yuying Children's Hospital of Wenzhou Medical University

Ren-Jun LV

Shandong Provincial Hospital Affiliated to Shandong First Medical University

Shan-Jing Nie

Shandong Provincial Hospital Affiliated to Shandong First Medical University

Lan-Bing Zhu

Second Affiliated Hospital \& Yuying Children's Hospital of Wenzhou Medical University

Xue-Ping Liu ( $\nabla$ Liuxueping1962@163.com )

Shandong University

\section{Research Article}

Keywords: Parkinson's disease, Polymorphism, Autophagy-related gene 7, Autophagy, Association

Posted Date: March 3rd, 2021

DOl: https://doi.org/10.21203/rs.3.rs-257405/v1

License: (9) (i) This work is licensed under a Creative Commons Attribution 4.0 International License. Read Full License 
$1 \quad$ ATG7 polymorphisms rs7625184 and rs2606750 are not associated with Parkinson's

2 disease: a case control study

3 Ming Zou ${ }^{\mathrm{a}, \mathrm{b}, \mathrm{c} \#}$, Jian-Yong Wang ${ }^{\mathrm{c}}$, Ren-Jun Lv ${ }^{\mathrm{b}}$, Shan-Jing Nie ${ }^{\mathrm{b}, \mathrm{d}, \mathrm{e}}$, Lan-Bing Zhu ${ }^{\mathrm{c}}$,

4 Xue-Ping $\operatorname{Liu}^{\mathrm{a}, \mathrm{b}, \mathrm{d}, \mathrm{e}^{*}}$

$5 \quad{ }^{\text {a }}$ Department of Geriatric Neurology, Shandong Provincial Hospital, Cheeloo College

6 of Medicine, Shandong University, Jinan, Shandong, 250021, China.

$7 \quad{ }^{\mathrm{b}}$ Department of Geriatric Neurology, Shandong Provincial Hospital Affiliated to

8 Shandong First Medical University, Jinan, Shandong, 250021, China.

$9 \quad{ }^{c}$ Department of Geriatrics \& Neurology, the Second Affiliated Hospital and Yuying

10 Children's Hospital, Wenzhou Medical University, Wenzhou, Zhejiang 325027, China

$11{ }^{\mathrm{d}}$ Anti-Aging Monitoring Laboratory, Shandong Provincial Hospital Affiliated to

12 Shandong First Medical University, Jinan, Shandong, 250021, China.

${ }^{\mathrm{e}}$ Department of Anti-Aging, Shandong Provincial Hospital Affiliated to Shandong

14 First Medical University, Jinan, Shandong, 250021, China.

15 \# These authors contribute equally to the work.

$16{ }^{*}$ Correspondence:

17 Xue-ping Liu, PhD, Department of Geriatric Neurology, Shandong Provincial

18 Hospital, Cheeloo College of Medicine, Shandong University, Jinan, Shandong,

19 250021, China; Tel: +8613455131453 ; Fax: +86053185187165; E-mail:

20 Liuxueping1962@163.com. 


\section{Abstract}

Background: Deregulation of autophagy is involved in the development and progression of Parkinson's disease. ATG7, an E1 like enzyme, palys a key role in autophagy. This study aimed to investigating the association between ATG7 polymorphisms and PD susceptibility.

Methods: Single nucleotide polymorphisms of ATG7, including rs7625184 and rs2606750, were identified by polymerase chain reaction-restriction fragment length polymorphism in a Han Chinese population consisting of 312 PD patients and 309 healthy controls.

Results: Genotyping analyses showed that none of the 2 SNPs was significantly associated with PD risk.

Conclusions: Our results suggest that rs7625184 and rs2606750 are not associated with PD susceptibility. Further studies are warranted in revealing the links between ATG7 and PD.

Keywords: Parkinson's disease, Polymorphism, Autophagy-related gene 7, Autophagy, Association 


\section{Background}

Parkinson's disease (PD) is the second most common neurodegenerative disease, that occurs in $1.7 \%$ of people over 65 in China [1]. PD is characterized by selectively loss of dopaminergic neurons and Lewy body formation in midbrain substantia nigra, and it is believed to be caused by many genetic and environmental factors [2, 3]. Alpha-synuclein $(\alpha$-syn), the main component of Lewy bodies, plays an important role in PD pathogenesis [4]. A growing body of evidence have linked $\alpha$-syn accumulation to the dysfunction of autophagy lysosomal pathway [5].

Autophagy is an essential degradation pathway for cell survival, which including three types: microautophagy, chaperone-mediated autophagy (CMA) and macroautophagy (hereafter called autophagy) [6]. Autophagy impairment generates dopamine neuron loss and $\alpha$-syn aggregation in substantia nigra [7]. Mutation of genes, such as Parkin, PINK1, ATP13A2 and FBXO7, that produce familial PD, have been found to be related with autophagy $[6,8]$.

The process of autophagy include initial steps, vesicle elongation, vesicle completion, membrane retrieval, docking and fusion, vesicle breakdown and degradation [9]. These events are regulated by proteins called autophagy-related genes (ATG). ATG7 is an E1 like enzyme, and it is a key molecule in vesicle elongation and vesicle completion processes [9]. Mouse models with deletion of ATG7 in midbrain dopamine neurons lead to reduced striatal dopamine content and ubiquitinated aggregate formation in neurons [10]. What's more, mice that lack ATG7 in nervous system presented a decreasing in coordinated movement [11].

To date, limited studies have been performed to investigate the association between ATG7 polymorphisms and PD [12, 13]. There was still no definitive conclusion if ATG7 single nucleotide polymorphisms (SNPs) are associated with PD risk. In this study, we aimed to explore whether ATG7 genetic variations are associated with PD susceptibility in a large Chinese cohort.

\section{Materials and methods}

\section{Subjects}


A total of 621 subjects of Han Chinese ethnicity Participated in this study, including 309 healthy controls (157 men and 152 women) and 312 sporadic PD patients (151 men and 161 women). The median age of PD patients and healthy controls were 67 (interquartile range: 60-75) and 70 (interquartile range: 57.5-78) years old respectively. The idiopathic PD patients were diagnosed according to the UK Parkinson's Disease Society Brain Bank Criteria by 2 movement disorder specialists [14]. Patients with secondly parkinsonism or with a family history of PD were excluded from our study. Healthy controls were free of neurological and psychotic disorders according to their medical history, physical and laboratory examinations. The PD and control groups were comparable by gender and age $(P=0.843$ and $P$ $=0.158$, respectively; Table 1$)$.

Table 1. Characteristics of the PD patients and controls

\begin{tabular}{lllll}
\hline & & Control & PD & $P$ \\
\hline Subject number & & 309 & 312 & \\
Gender & Male, n (\%) & $157(50.8)$ & $151(48.4)$ & $0.843^{\mathrm{a}}$ \\
& Female, $\mathrm{n}(\%)$ & $152(49.2)$ & $161(51.6)$ & \\
Age median (IR) & & $70(57.5-78)$ & $67(60-75)$ & $0.158^{\mathrm{b}}$ \\
\hline
\end{tabular}
PD, Parkinson's disease; IR, interquartile range.

${ }^{\text {a }}$ Analyzed by Chi square test.

${ }^{\mathrm{b}}$ Analyzed by Mann-Whitney Test.

\section{Single nucleotide polymorphisms (SNPs)}

Thirteen tag-single nucleotide polymorphisms (tag-SNPs) of ATG7, including rs11707842, rs7625184, rs2454476, rs2606750, rs2447607, rs2594992, rs17034276, rs2305295, rs4684776, rs4684787, rs6442260, rs9818393, and rs9873812, were identified according to the HapMap project and Haploview v.4.2 [15]. The parameters are as follows: $\mathrm{r} 2 \geq 0.8$, and mean allele frequency $(\mathrm{MAF}) \geq 0.1$ in Han Chinese 
110 Table 2. PCR primers and RFLP products

\begin{tabular}{lllll}
\hline SNPs & Restriction enzyme & Primers & PCR product, bp & RFLP size, bp \\
\hline rs7625184 & AccI & Forward: 5'-GCATAATCTTACCACTGG -3' & 561 & TT: 561 \\
& & Reverse: 5'-CTCTCCATTCCCACTGCTAC -3' & & TC: $561+497+64$ \\
& & & CC: $497+64$ \\
rs2606750 & \multirow{2}{*}{ BstBI } & Forward: 5'-AAGACTTGTCCCTTCACATT -3' & 532 & TT: 532 \\
& & Reverse: 5'-CCTTTCCCATCCCACTCCA -3' & TC: $532+358+174$ \\
& & & CC: $358+174$ \\
\hline
\end{tabular}

PCR, polymerase chain reaction; RFLP, restriction fragment length polymorphism; SNPs, single nucleotide polymorphisms.

\section{Data analysis}

116 All of the analyses in our study were performed by using statistical package of

117 Predictive Analytics Software 18.0 (PASW, version 18.0) for windows. The

population from Beijing, China. We finally selected rs7625184 (T>C) and rs2606750 $(\mathrm{T}>\mathrm{C})$ in our study, because both of them could be digested by restriction enzymes, when their allele is " $\mathrm{C}$ ".

\section{Genotyping}

Genomic DNA was extracted from the peripheral blood samples of participants using a DNA blood kit (Tiangen, Beijing, China), as described before [16]. SNPs were determined by polymerase chain reaction-restriction fragment length polymorphism (PCR-RFLP) analysis. The primer pairs, restriction enzymes, and fragment length of SNPs were presented in table 2. PCRs were conducted according to the manufacturer's protocol (Tiangen, Beijing, China). The annealing temperature were $52^{\circ} \mathrm{C}$ for rs 7625184 , and $56^{\circ} \mathrm{C}$ for $\mathrm{rs} 2606750$. PCR products were digested by restriction endonucleases according to manufacturer's protocol (New England BioLabs, Beverly, MA; Table 2). The digested fragments were separated and visualized as described previously [16]. Twenty PCR samples from each SNPs were verified by direct sequencing (BGI Tech, Shanghai, China), and all of them were consistent with the enzymatic genotyping. 
119 to evaluate the genotype distribution of the population and normality respectively. The

120 differences in gender, and genotype and allele frequencies between PD and control

121 groups were assessed by $\chi^{2}$ test. The difference in age between the two groups was

122 assessed by Mann-Whitney Test. Multivariate analysis was performed by binary

123 logistic regression model with gender, age and genotypes as covariates. A two-tailed $P$

124 value $<0.05$ was considered statistically significant.

\section{Results}

126 The ATG7 variants, rs7625184 and rs2606750 were not associated with PD

\section{7 susceptibility}

128 The genotype distribution of rs7625184 and rs2606750 in PD patients and healthy 129 controls met with HWE $(P>0.05)$. For both rs7625184 and rs2606750, no statistical

130 difference in genotype distribution was found between PD and controls groups $(P$

$131=0.904$ and $P=0.280$, respectively; Table 3 ). There was also no statistical difference

132 in their allele frequencies between the 2 groups ( $P=0.659$ and $P=0.128$, respectively;

133 Table 3). We further performed a logistic regression analysis with gender, age, and the

134 two SNPs as covariates. The result showed that neither rs7625184 nor rs2606750 was

135 risk factor for PD (Table 4).

136

137 Table 3. Genotype and allele frequencies of rs7625184 and rs2606750 in PD patients and controls

\begin{tabular}{|c|c|c|c|c|c|c|c|c|}
\hline \multirow{2}{*}{$\begin{array}{l}\text { SNPs } \\
\text { rs7625184 }\end{array}$} & \multicolumn{3}{|c|}{ Genotype, n (\%) } & \multirow[t]{2}{*}{$P$} & \multicolumn{2}{|c|}{ Allele, n (\%) } & \multirow[t]{2}{*}{$P$} & \multirow[t]{2}{*}{ OR $(95 \% \mathrm{CI})$} \\
\hline & TT & $\mathrm{TC}$ & $\mathrm{CC}$ & & $\mathrm{T}$ & $\mathrm{C}$ & & \\
\hline Controls & $153(49.5)$ & $127(41.1)$ & $29(9.4)$ & 0.904 & $433(70.1)$ & 185 (29.9) & 0.659 & $1.056(0.829-1.345)$ \\
\hline PD & $149(47.8)$ & $132(42.3)$ & $31(9.9)$ & & $430(68.9)$ & $194(31.1)$ & & \\
\hline rs 2606750 & $\mathrm{TT}$ & $\mathrm{TC}$ & $\mathrm{CC}$ & & $\mathrm{T}$ & $\mathrm{C}$ & & \\
\hline Controls & 43 (13.9) & $162(52.4)$ & $104(33.7)$ & 0.280 & $248(40.1)$ & $370(59.9)$ & 0.128 & $0.840(0.670-1.052)$ \\
\hline PD & $54(17.3)$ & $169(54.2)$ & $89(28.5)$ & & $277(44.4)$ & $347(55.6)$ & & \\
\hline
\end{tabular}

138 CI, confidence interval; OR, odds ratio; PD, Parkinson's disease; SNPs, single nucleotide

139 polymorphisms.

141 Table 4. Multivariate analysis of risk factors for PD by binary logistic regression

\begin{tabular}{|c|c|c|c|c|c|}
\hline \multirow{2}{*}{ Factors } & \multirow{2}{*}{ B } & \multirow{2}{*}{$P$} & \multirow{2}{*}{ OR } & \multicolumn{2}{|c|}{$95 \% \mathrm{CI}$ for $\mathrm{OR}$} \\
\hline & & & & Lower & Upper \\
\hline
\end{tabular}

$\begin{array}{llll}\text { Constant } & 0.010 & 0.904 & 1.010\end{array}$

$143{ }^{a}$ Binary logistic regression with gender, age, and 2 SNPs as covariates. 


\section{Discussion}

Accumulating evidence showed that autophagy dysfunction plays an essential role in $\alpha$-syn degradation and PD pathology [5, 17-19]. ATG7 is a key enzyme in autophagy pathway $[6,9,20]$, and it is associated with PD and dementia with lewy bodies (DLB) $[10,11,21,22]$. In the present study, we performed a case-control study to investigate the relationship between ATG7 SNPs and PD risk in a currently largest cohort. However, our results showed that both rs7625184 and rs2606750 were not associated with PD susceptibility.

Both rs7625184 and rs2606750 are located in intron of ATG7. There was no known clinical case has been reported relating to these two sites. In our cohort, $\mathrm{C}$ allele of rs7625184 and $\mathrm{T}$ allele of rs2606750 are the minor alleles $(30.5 \%$ and 42.3\%). It is in accordance with the frequency of Asian population in NCBI dbSNP (35.0\% and 17.0\%). As far as we know, our research is the first attempt to explore the relationship between the two SNPs and PD, though we got a negative result.

Two previous studies have been carried out to analyse the association between ATG7 SNPs and sporadic PD. Chen et al. sequenced ATG7 promotor region in 101 PD patients and 148 healthy controls. They identified four novel heterozygous variants $(11313449 \mathrm{G}>\mathrm{A}, 11313811 \mathrm{~T}>\mathrm{C}, 11313913 \mathrm{G}>\mathrm{A}$ and $11314041 \mathrm{G}>\mathrm{A})$ in $\mathrm{PD}$ patients, and found that these mutations decreased transcriptional activities of the ATG7 gene promoter by luciferase reporter [13]. However, due to the limited sample size, it remains unclear whether the four mutations affect the autophagic activity and PD susceptibility. The other study analyzed rs1375206 (a SNP in promoter) and plasma ATG7 levels in 124 PD patients and 105 comparable healthy controls. They found the plasma ATG7 levels were higher in PD patients, but no significant difference in genotype distribution was found between two groups [12]. Further studies should be performed to elucidate the association between ATG7 SNPs and PD susceptibility.

As we know, dysfunctions in autophagy have been observed in Huntington disease and PD[23]. ATG7 polymorphism (V471A) has been identified to be related 
with age at onset of Huntington's disease [23, 24]. In addition, deletion of ATG7 has been widely used in PD research as an autophagy model $[25,26]$. And it has also been explored as a potential drug therapy target of PD [27-29]. Therefore, it is of great significance to further explore the relationship between ATG7 and PD, as well as the underlying mechanism.

However, in our study, we selected rs7625184 and rs2606750 instead of all the tag-SNPs, because they could be digested by restriction enzymes. This may limited the objectivity of our conclusion. Future investigations are warranted to further uncover the association between ATG7 and PD.

\section{Conclusions}

In conclusion, the current study suggest in a Chinese cohort that rs7625184 and rs2606750 of ATG7 were not associated with PD susceptibility. Further studies are needed to define the role of ATG7 in PD as well as the causality of the polymorphisms.

\section{List of abbreviations}

ATG: Autophagy-related genes; CMA: Chaperone-mediated autophagy; DLB: Dementia with lewy bodies; HWE: Hardy-Weinberg equilibrium; KS: Kolmogorov-Smirnov; MAF: Mean allele frequency; PCR: polymerase chain reaction; PCR-RFLP: Polymerase chain reaction-restriction fragment length polymorphism; PD: Parkinson's disease; SNPs: Single nucleotide polymorphisms

\section{Ethics approval and consent to participate}

The study was approved by the Ethics Committee of The Second Affiliated Hospital and Yuying Children's Hospital, Wenzhou Medical University. All subjects signed written informed consents prior to participation in the study. All methods were carried out in accordance with relevant guidelines and regulations.

\section{Availability of data and materials}

The data that support the findings of this study are available from the corresponding 
author upon reasonable request.

\section{Competing interests}

The authors declare that there is no potential conflict of interest.

\section{Funding}

The study was supported in part by funding from National Natural Science Foundation of China (81801271), and Wenzhou Municipal Science and Technology Bureau (Y2020065).

\section{Acknowledgements}

The authors are grateful to all of the subjects for participating in this study.

\section{Authors' contributions}

MZ, JYW and XPL designed the study. MZ, JYW and LBZ examined the patients, and collected blood samples. JYW and LBZ analyzed blood samples and interpreted the genetic data. RJL and SJN provided statistical support. XPL and JYW drafted the manuscript. XPL supervised the study. All authors read, revised and approved the final version of the manuscript.

\section{References}

1. Zhang ZX, Roman GC, Hong Z, Wu CB, Qu QM, Huang JB, et al. Parkinson's disease in China: prevalence in Beijing, Xian, and Shanghai. Lancet. 2005;365:595-7.

2. Greenamyre JT, Hastings TG. Biomedicine. Parkinson's--divergent causes, convergent mechanisms. Science. 2004;304:1120-2.

3. McCulloch CC, Kay DM, Factor SA, Samii A, Nutt JG, Higgins DS, et al. 
231 Exploring gene-environment interactions in Parkinson's disease. Human genetics. $232 \quad 2008 ; \mathbf{1 2 3}: 257-65$.

233 4. Luk KC, Kehm V, Carroll J, Zhang B, O'Brien P, Trojanowski JQ, et al. 234 Pathological alpha-synuclein transmission initiates Parkinson-like 235 neurodegeneration in nontransgenic mice. Science. 2012;338:949-53.

236 5. Xilouri M, Brekk OR, Stefanis L. Autophagy and Alpha-Synuclein: Relevance to Parkinson's Disease and Related Synucleopathies. Mov Disord. 2016;31:178-92. 6. Karabiyik C, Lee MJ, Rubinsztein DC. Autophagy impairment in Parkinson's disease. Essays Biochem. 2017;61:711-20.

7. Hunn BHM, Vingill S, Threlfell S, Alegre-Abarrategui J, Magdelyns M, Deltheil $\mathrm{T}$, et al. Impairment of Macroautophagy in Dopamine Neurons Has Opposing Effects on Parkinsonian Pathology and Behavior. Cell Rep. 2019;29:920-31 e7. 8. Gusdon AM, Zhu J, Van Houten B, Chu CT. ATP13A2 regulates mitochondrial bioenergetics through macroautophagy. Neurobiol Dis. 2012;45:962-72. 9. Levine B, Kroemer G. SnapShot: Macroautophagy. Cell. 2008;132:162 e1- e3. 10. Friedman LG, Lachenmayer ML, Wang J, He L, Poulose SM, Komatsu M, et al. Disrupted autophagy leads to dopaminergic axon and dendrite degeneration and promotes presynaptic accumulation of alpha-synuclein and LRRK2 in the brain. J Neurosci. 2012;32:7585-93. autophagy in the central nervous system causes neurodegeneration in mice. Nature. 2006;441:880-4.

12. Zhao X, Chen Y, Wang L, Li X, Chen X, Zhang H. Associations of ATG7 rs1375206 polymorphism and elevated plasma ATG7 levels with late-onset sporadic Parkinson's disease in a cohort of Han Chinese from southern China. Int J Neurosci. 2020:1-9. ATG7 gene promoter in sporadic Parkinson's disease. Neurosci Lett. 2013;534:193-8. 
idiopathic Parkinson's disease: a clinico-pathological study of 100 cases. Journal of neurology, neurosurgery, and psychiatry. 1992;55:181-4.

15. Barrett JC, Fry B, Maller J, Daly MJ. Haploview: analysis and visualization of LD and haplotype maps. Bioinformatics. 2005;21:263-5.

16. Zhang X, Cheng X, Hu YB, Lai JM, You H, Hu PL, et al. Serotonin transporter polymorphic region 5-HTTLPR modulates risk for Parkinson's disease. Neurobiol Aging. 2014;35:1957 e9- e14.

17. Cuervo AM, Stefanis L, Fredenburg R, Lansbury PT, Sulzer D. Impaired degradation of mutant alpha-synuclein by chaperone-mediated autophagy. Science. 2004;305:1292-5.

18. Pan T, Kondo S, Le W, Jankovic J. The role of autophagy-lysosome pathway in neurodegeneration associated with Parkinson's disease. Brain. 2008;131:1969-78. 19. Webb JL, Ravikumar B, Atkins J, Skepper JN, Rubinsztein DC. Alpha-Synuclein is degraded by both autophagy and the proteasome. The Journal of biological chemistry. 2003;278:25009-13.

20. Ichimura Y, Imamura Y, Emoto K, Umeda M, Noda T, Ohsumi Y. In vivo and in vitro reconstitution of Atg8 conjugation essential for autophagy. The Journal of biological chemistry. 2004;279:40584-92.

21. Crews L, Spencer B, Desplats P, Patrick C, Paulino A, Rockenstein E, et al. Selective molecular alterations in the autophagy pathway in patients with Lewy body disease and in models of alpha-synucleinopathy. PLoS One. 2010;5:e9313.

22. Sato S, Hattori N. Dopaminergic Neuron-Specific Autophagy-Deficient Mice. Methods Mol Biol. 2018;1759:173-5.

23. Metzger S, Saukko M, Van Che H, Tong L, Puder Y, Riess O, et al. Age at onset in Huntington's disease is modified by the autophagy pathway: implication of the V471A polymorphism in Atg7. Human genetics. 2010;128:453-9.

24. Metzger S, Walter C, Riess O, Roos RA, Nielsen JE, Craufurd D, et al. The V471A polymorphism in autophagy-related gene ATG7 modifies age at onset specifically in Italian Huntington disease patients. PLoS One. 2013;8:e68951.

25. Zhou J, Song J, Wu S. Autophagic degradation of stromal interaction 
291 molecule 2 mediates disruption of neuronal dendrites by endoplasmic reticulum 292 stress. J Neurochem. 2019;151:351-69.

293 26. Ahmed I, Liang Y, Schools S, Dawson VL, Dawson TM, Savitt JM.

294 Development and characterization of a new Parkinson's disease model resulting 295 from impaired autophagy. J Neurosci. 2012;32:16503-9.

296 27. Niu XY, Huang HJ, Zhang JB, Zhang C, Chen WG, Sun CY, et al. Deletion of 297 autophagy-related gene 7 in dopaminergic neurons prevents their loss induced by 298 MPTP. Neuroscience. 2016;339:22-31.

299 28. Xie C, Ginet V, Sun Y, Koike M, Zhou K, Li T, et al. Neuroprotection by 300 selective neuronal deletion of Atg7 in neonatal brain injury. Autophagy. $301 \quad 2016 ; 12: 410-23$.

302 29. Han B, Wang L, Fu F, Wang Z, Zhang L, Qi GJ, et al. Hydroxysafflor yellow A 303 promotes alpha-synuclein clearance via regulating autophagy in 304 rotenone-induced Parkinson's disease mice. Folia Neuropathol. 2018;56:133-40. 